\title{
Identification of a limiting mechanism in GaSb-rich superlattice midwave infrared detector
}

Marie Delmas, Jean-Baptiste Rodriguez, Rémi Rossignol, Abigail S. Licht, Edouard Giard, Isabelle RibetMohamed, and Philippe Christol

Citation: Journal of Applied Physics 119, 174503 (2016);

View online: https://doi.org/10.1063/1.4948670

View Table of Contents: http://aip.scitation.org/toc/jap/119/17

Published by the American Institute of Physics

\section{Articles you may be interested in}

Electrical modeling of InAs/GaSb superlattice mid-wavelength infrared pin photodiode to analyze experimental dark current characteristics

Journal of Applied Physics 116, 113101 (2014); 10.1063/1.4895983

Quantum efficiency investigations of type-II InAs/GaSb midwave infrared superlattice photodetectors

Journal of Applied Physics 116, 043101 (2014); 10.1063/1.4890309

Dark current suppression in type II InAs / GaSb superlattice long wavelength infrared photodiodes with Mstructure barrier

Applied Physics Letters 91, 163511 (2007); 10.1063/1.2800808

Extended short-wavelength infrared $\mathrm{nBn}$ photodetectors based on type-II InAs/AISb/GaSb superlattices with an $\mathrm{AlAsSb} / \mathrm{GaSb}$ superlattice barrier

Applied Physics Letters 110, 101104 (2017); 10.1063/1.4978378

InAs/GaSb type-II superlattice infrared detectors: Future prospect

Applied Physics Reviews 4, 031304 (2017); 10.1063/1.4999077

$n B n$ detector, an infrared detector with reduced dark current and higher operating temperature

Applied Physics Letters 89, 151109 (2006); 10.1063/1.2360235

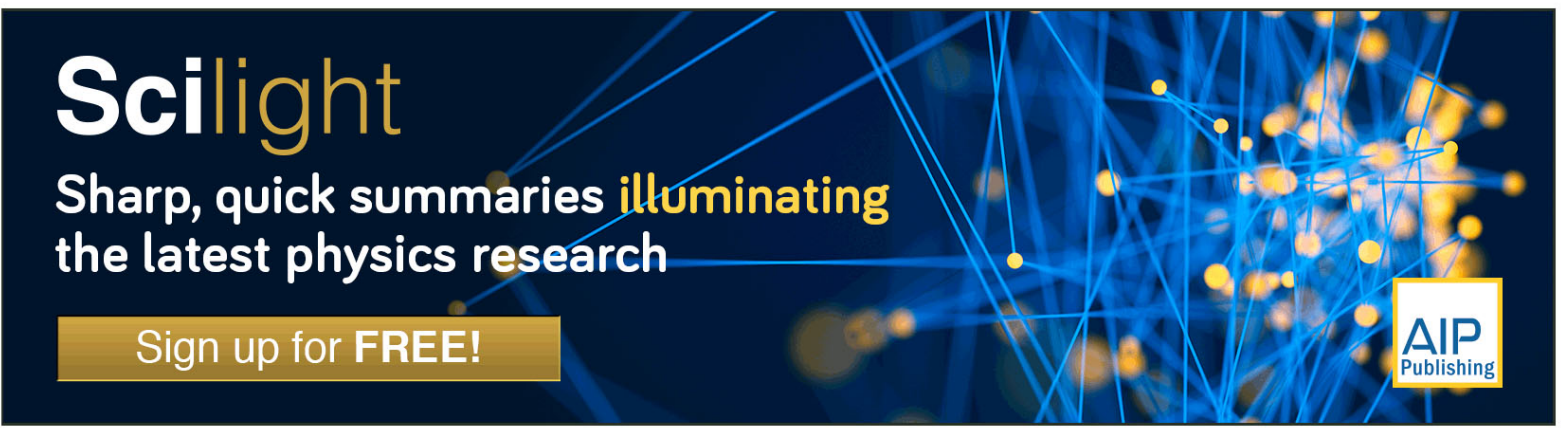




\title{
Identification of a limiting mechanism in GaSb-rich superlattice midwave infrared detector
}

\author{
Marie Delmas, ${ }^{1,2}$ Jean-Baptiste Rodriguez, ${ }^{1,2}$ Rémi Rossignol, ${ }^{1,2}$ Abigail S. Licht, ${ }^{1,2,3}$ \\ Edouard Giard, ${ }^{4}$ Isabelle Ribet-Mohamed, ${ }^{4}$ and Philippe Christol ${ }^{1,2, a)}$ \\ ${ }^{1}$ Université de Montpellier, IES, UMR 5214, F-34000 Montpellier, France \\ ${ }^{2}$ CNRS, IES, UMR 5214, F-34000 Montpellier, France \\ ${ }^{3}$ Renewable Energy and Applied Photonics Laboratories, Electrical and Computer Engineering Department, \\ Tufts University, Medford, Massachusetts 02155, USA \\ ${ }^{4}$ ONERA, Chemin de la Hunière, 91761 Palaiseau, France
}

(Received 17 February 2016; accepted 23 April 2016; published online 6 May 2016)

GaSb-rich superlattice (SL) p-i-n photodiodes grown by molecular beam epitaxy were studied theoretically and experimentally in order to understand the poor dark current characteristics typically obtained. This behavior, independent of the SL-grown material quality, is usually attributed to the presence of defects due to Ga-related bonds, limiting the SL carrier lifetime. By analyzing the photoresponse spectra of reverse-biased photodiodes at $80 \mathrm{~K}$, we have highlighted the presence of an electric field, breaking the minibands into localized Wannier-Stark states. Besides the influence of defects in such GaSb-rich SL structures, this electric field induces a strong tunneling current at low bias which can be the main limiting mechanism explaining the high dark current density of the GaSb-rich SL diode. Published by AIP Publishing.

[http://dx.doi.org/10.1063/1.4948670]

\section{INTRODUCTION}

Among the high performance cooled infrared (IR) photodetectors, the InAs/GaSb superlattice (SL) material system is an emerging competitor. ${ }^{1}$ The particular type-II broken gap band alignment of the InAs/GaSb SL structure provides many advantages for IR detection. Through modulating the periodicity of the constituent SL ratios, the band structure can be engineered to a specific wavelength over a wide range of values while maintaining the SL structure lattice matched to the GaSb substrate ${ }^{2}$ and reducing the dark current through the management of Auger recombination rates. ${ }^{3}$ However, concerning dark currents, the InAs/GaSb SL has not yet reached its theoretical predictions nor surpassed those of mercury cadmium telluride (MCT) detectors. ${ }^{4,5}$ The main reason is that the Shockley-Read-Hall (SRH) mechanism limits the minority carrier lifetime of the SL, which results in higher current densities than those of MCT. In the midwave infrared (MWIR) and longwave infrared (LWIR) spectral domain, the measured SL minority carrier lifetimes range from $10 \mathrm{~ns}$ to about $150 \mathrm{~ns}$ at $77 \mathrm{~K},{ }^{6-14}$ which are significantly lower than MCT carrier lifetimes. ${ }^{9}$ For many years, the poor carrier lifetimes in SL materials have been attributed to native defects in the GaSb binary layer and other Ga-related bonds. ${ }^{10,11}$ As a result, the InAs/InAsSb SL (called "GaFree") has been considered as an alternative to the InAs/ $\mathrm{GaSb}$ SL because of the longer carrier lifetimes of this material in the MWIR ${ }^{15}$ and LWIR. ${ }^{16}$ Despite this fact, Ga-Free detector performances have not proved to be superior to InAs/GaSb detectors. ${ }^{17,18}$

\footnotetext{
a) Author to whom correspondence should be addressed. Electronic mail: christol@ies.univ-montp2.fr. Tel.: (+33) 467143826.
}

For a better understanding of the impact of the GaSb content, we have recently studied the influence of the InAs/ GaSb SL period composition and the thickness on the material and device properties. ${ }^{19,20}$ We found that the SL period mainly composed of $\mathrm{GaSb}$ (hereto referred to as "GaSbrich") has a higher current density than the SL structure mainly composed of InAs ("InAs-rich") having the same cut-off wavelength of $5 \mu \mathrm{m}$ at $77 \mathrm{~K}$. The current density difference between these MWIR structures is extremely large with almost a factor of 50 .

In this work, the GaSb-rich SL structure is further studied in order to understand the poor dark current characteristics typically obtained compared to the InAs-rich structures. Section II explores the influence of the growth conditions of the GaSb-rich SL structure on the electrical performance. The dark current is then analyzed in Section III with ATLAS simulation software from SILVACO. In Section IV, spectral photoresponses of the GaSb-rich SL structures under electric field are reported, highlighting the main mechanism limiting the electrical performances of MWIR GaSb-rich SL photodiodes.

\section{INFLUENCE OF THE GROWTH CONDITIONS ON ELECTRICAL PERFORMANCES}

Although the minority carrier lifetime can be lower in the GaSb-rich SL structure than in the InAs-rich one,${ }^{10}$ it cannot itself account for the large difference in current density values. ${ }^{19}$ Nevertheless, a possibility is that the molecular beam epitaxy (MBE) growth conditions used for the InAs/ GaSb SL are not suitable for the GaSb-rich SL, resulting in extremely low lifetimes. The SL growth temperature is usually $400{ }^{\circ} \mathrm{C}$ in order to maintain a good quality of the InSblike interfaces. ${ }^{21}$ However, the GaSb binary compound is 
usually grown at higher temperature than $400{ }^{\circ} \mathrm{C}$. The optimal growth temperature of the GaSb-rich period could therefore also be higher. As such, we studied higher growth temperatures $\left(450^{\circ} \mathrm{C}\right)$ of $\mathrm{GaSb}$-rich $\mathrm{SL}$ in order to improve the current density values. Because the InSb-like interfaces are degraded at $450{ }^{\circ} \mathrm{C}$, the shutter sequences employed during the growth were first adapted to grow the GaSb-rich SL structures at high temperatures.

We then compared the electrical performances of two similar GaSb-rich SL p-i-n photodiodes grown at different substrate temperatures $\left(\mathrm{T}_{\text {growth }}\right)$ in a RIBER Compact 21 MBE reactor. Sample A was grown at $450{ }^{\circ} \mathrm{C}$ with optimized growth conditions for high temperature and sample B at $400{ }^{\circ} \mathrm{C}$ using usual growth conditions. ${ }^{21}$ The GaSb-rich SL p-i-n structures studied consist of a $200 \mathrm{~nm}$ thick p-doped $\left(\mathrm{P} \sim 1 \times 10^{18} \mathrm{~cm}^{-3}\right) \mathrm{GaSb}$ buffer layer, followed by a $60 \mathrm{~nm}$ thick p-doped $\left(\mathrm{P} \sim 1 \times 10^{18} \mathrm{~cm}^{-3}\right) \mathrm{InAs} / \mathrm{GaSb} \mathrm{SL}$, a $500 \mathrm{~nm}$ thick non-intentionally doped (nid) $\mathrm{InAs} / \mathrm{GaSb} \mathrm{SL}$ active region, a $60 \mathrm{~nm}$ thick n-doped $\left(\mathrm{N} \sim 5 \times 10^{17} \mathrm{~cm}^{-3}\right)$ InAs/ GaSb SL, and a $20 \mathrm{~nm}$ Te-doped $\left(\mathrm{N} \sim 5 \times 10^{17} \mathrm{~cm}^{-3}\right)$ InAs contact layer. The InAs/GaSb SL period of the active region is composed of 11 monolayers (MLs) of InAs and 20 MLs of $\mathrm{GaSb}$, for a cut-off wavelength at around $4.75 \mu \mathrm{m}$ at $77 \mathrm{~K}$.

To validate the structural quality of the two samples, the surface morphology has been observed by the atomic force microscope (AFM) on a $2 \mu \mathrm{m} \times 2 \mu \mathrm{m}$ scan area (Figure 1). Excellent root mean square (RMS) surface roughness values were obtained and atomic steps were clearly observed.

Circular mesa photodiodes were then fabricated using standard photolithography procedures. Mesa photodiodes were realized by wet-etching and the polymerized photoresist was subsequently spread onto the surface to protect the samples from ambient air. ${ }^{22}$

Current density voltage characteristics were measured using a Keithley 6571A electrometer. The current density of samples A and B under dark conditions at $77 \mathrm{~K}$ is reported in Figure 2. As a bench mark comparison, the dark current density of an InAs-rich SL pin photodiode, having a cut-off wavelength of $5.15 \mu \mathrm{m}$ at $77 \mathrm{~K},{ }^{23}$ is also plotted. It can be seen that the reverse dark current for both GaSb-rich structures is close. While the growth conditions were optimized at high temperature, the dark current of the GaSb-rich photodiode has not been improved. Despite the very different growth conditions (substrate temperature, shutter sequence), the two devices show similar dark current density, which implies that the limiting factor is unlikely to be the material quality as initially suggested. a)

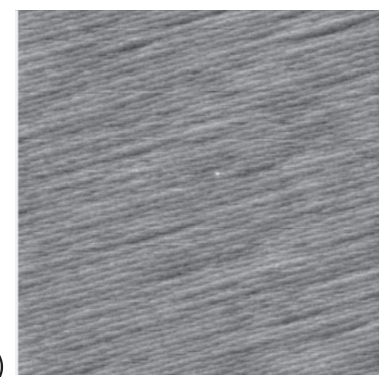

b)

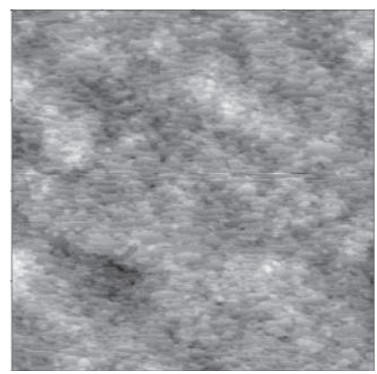

FIG. 1. AFM on a $2 \mu \mathrm{m} \times 2 \mu \mathrm{m}$ scan area: sample A (a) and sample B (b).

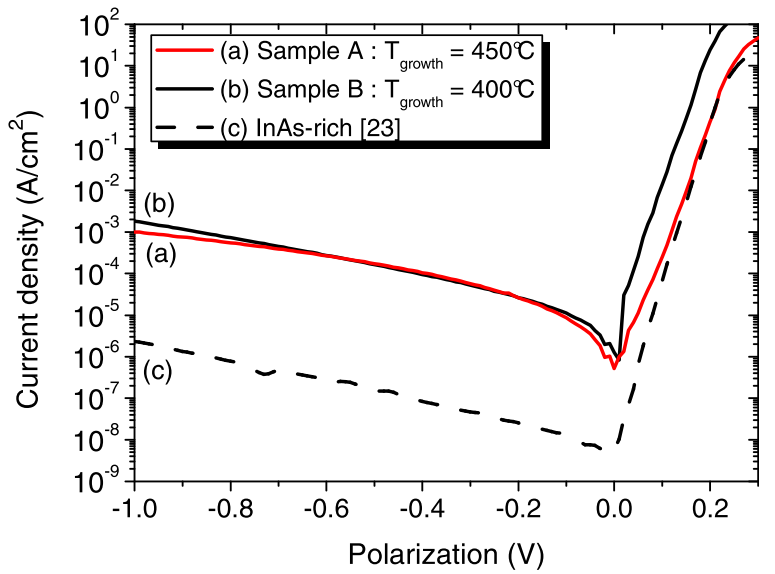

FIG. 2. Current density as function of the applied voltage of sample A $\left(\mathrm{T}_{\text {growth }}=450{ }^{\circ} \mathrm{C}\right)$ and sample $\mathrm{B}\left(\mathrm{T}_{\text {growth }}=400^{\circ} \mathrm{C}\right)$ performed in dark conditions at $77 \mathrm{~K}$. As comparison, the current density of an InAs-rich SL photodiode is also plotted (dashed line). ${ }^{23}$

In order to better understand these results, simulations of the dark current were performed using the TCAD (Technology Computer Aided Design) SILVACO software and the results are presented in Section III.

\section{DARK CURRENT SIMULATION}

The simulations reported in this section were done using the ATLAS software from SILVACO which has already been used to simulate the dark current of InAs-rich SL p-i-n photodiodes. ${ }^{23}$ To determine the dark current-voltage characteristics of the MWIR GaSb-rich SL photodiode, a bulkbased model with the effective band gap of the SL material $(261 \mathrm{meV}$ at $77 \mathrm{~K})$ is used. For the dark current density of the diode $\left(\mathrm{J}_{\text {diode }}\right)$, several contributions have to be considered: diffusion current $\left(\mathrm{J}_{\text {Diff }}\right)$, generation-recombination current $\left(\mathrm{J}_{\mathrm{GR}}\right)$, trap assisted tunneling current $\left(\mathrm{J}_{\mathrm{TAT}}\right)$, and bandto-band tunneling current $\left(\mathrm{J}_{\mathrm{BTB}}\right)$. The total dark current of the diode is then given by

$$
\mathrm{J}_{\text {diode }}=\mathrm{J}_{\text {Diff }}+\mathrm{J}_{\mathrm{GR}}+\mathrm{J}_{\mathrm{TAT}}+\mathrm{J}_{\mathrm{BTB}} .
$$

The material parameters of the GaSb-rich SL have to be specified for the dark current simulation. Some parameters, such as the effective electron mass ${ }^{24}$ were found in the literature, while the others were set by calculating the weighted average of InAs and GaSb bulk values available in the ATLAS manual ${ }^{25}$ and extracted from Ref. 26 . The conductivity of the minority carriers was determined by the Hall measurement. A dedicated nid GaSb-rich SL structure was grown by MBE on GaSb substrate. Next, a specific process was done to remove the conductive GaSb substrate and then the Hall measurement was performed at $77 \mathrm{~K}$, giving a p-type conductivity. More details on such measurements can be found in Ref. 27. The background carrier concentration was extracted from the capacitance-voltage characteristic of a GaSb-rich SL p-i-n diode. A residual carrier concentration equal to $4.3 \times 10^{15} \mathrm{~cm}^{-3}$ was determined at $77 \mathrm{~K}$ for sample A. The material parameters of the GaSb-rich SL chosen for the simulation are summarized in Table I. 
TABLE I. Parameter values used for GaSb-rich SL in ATLAS simulation. ${ }^{25,26}$

\begin{tabular}{lc}
\hline \hline Parameter & Values \\
\hline Varshni's parameter $\alpha$ & $0.35 \mathrm{meV} / \mathrm{K}$ \\
Varshni's parameter $\beta$ & $350 \mathrm{~K}$ \\
Permittivity & $15.50 \varepsilon_{0}$ \\
Electron affinity & $4.63 \mathrm{eV}$ \\
Electron effective mass & $0.0382 \mathrm{~m}_{0}$ \\
Hole effective mass & $0.236 \mathrm{~m}_{0}$ \\
\hline \hline
\end{tabular}

In this section, we analyze the dark current of sample A. Although it has particular growth conditions, its dark current is of the same order of magnitude than the GaSb-rich SL photodiodes grown under standard growth conditions.

The model used for the dark current simulations is the same as the one used in previous publications. ${ }^{23}$ In particular, we took into account the generation-recombination and the trap-assisted tunneling current through the Hurkx model. ${ }^{28}$ The trap energy level, the tunneling mass, and the minority carrier lifetime are the parameters to be determined to fit the experimental curve. The minority carrier lifetime of the GaSb-rich SL structure was extracted from time resolved photoluminescence (TRPL) measurements. These experiments were done on a dedicated sample made of nid GaSbrich SL structure $(500 \mathrm{~nm}$ thick) sandwiched by two nid AlSb barriers (20 nm thick) to confine the optically injected carriers into the SL region (inset of Figure 3). An optical parametric oscillator (OPO) light source at $1.55 \mu \mathrm{m}$ was used as an excitation source, with a pulse width of $10 \mathrm{~ns}$ and a repetition rate of $149.8 \mathrm{kHz}$. The PL signal was focused on a fast $\mathrm{HgCdTe}$ single pixel detector ( $8 \mu \mathrm{m}$ cut-off wavelength, $4 \mathrm{~ns}$ temporal response, and $200 \mathrm{~K}$ operating temperature), and a $2.5-20 \mu \mathrm{m}$ band-pass filter was used to avoid any residual light collection. The PL decays were then sampled by a digital oscilloscope.

Figure 3 presents the photoluminescence signal $\left(\mathrm{I}_{\mathrm{PL}}\right)$ as a function of time for sample A at $80 \mathrm{~K}$. We observe an exponential decay which can be fitted by the following equation: ${ }^{8}$

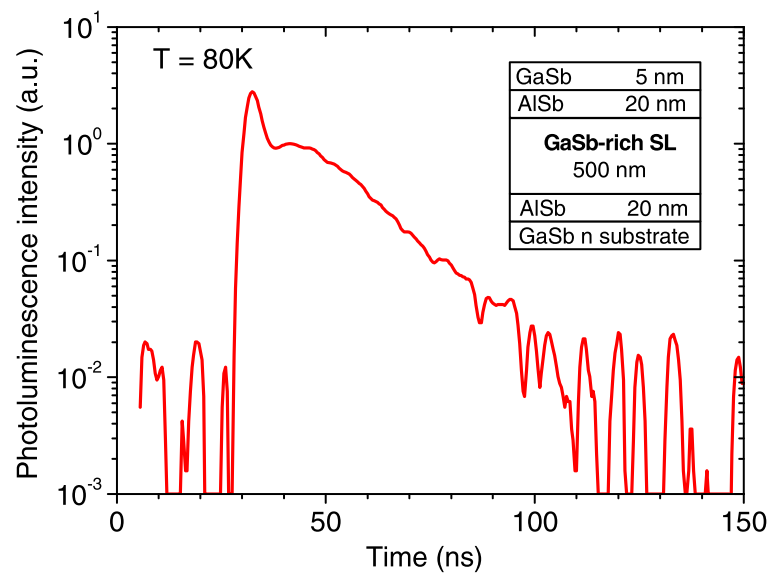

FIG. 3. Experimental time-resolved photoluminescence data performed at $80 \mathrm{~K}$ on a dedicated structure grown on n-type GaSb substrate (inset). The fast initial peak decay is due to optical-injection of excess carriers.

$$
I_{P L}(t)=A \cdot \delta_{n_{0}}^{2} \cdot\left[e^{-2 t / \tau}+\frac{p}{\delta_{n_{0}}} e^{-t / \tau}\right]
$$

where $A$ is an experimental constant, $p$ is the residual background concentration, estimated equal to $4.3 \times 10^{15} \mathrm{~cm}^{-3}$, $\delta_{\mathrm{n}_{0}}$ is the optically generated excess carrier density at $t=0 \mathrm{~s}$, estimated equal to $7 \times 10^{17} \mathrm{~cm}^{-3}$, and $\tau$ is the minority carrier lifetime.

Using a Metropolis-Hastings algorithm, the minority carrier lifetime was determined to be equal to $30 \mathrm{~ns}$ at $80 \mathrm{~K}$ which is consistent with recent measurements performed on specific MWIR SL samples. ${ }^{12}$

The trap energy level was fixed to the Fermi energy level, where they are the most efficient. Finally, the tunneling mass was calculated as the reduced mass equal to $\left(m_{c} \times m_{h}\right) /\left(m_{c}+m_{h}\right)$, with $m_{c}$ and $m_{h}$ being the electron and hole effective mass, respectively.

The experimental and simulated dark currents using these parameters are plotted in Figure 4. We observe in reverse bias a large difference of about two orders of magnitude between both curves. The fitting can be improved by decreasing the lifetime down to $0.3 \mathrm{~ns}$ rather than $30 \mathrm{~ns}$ (Figure 4). In this case, a good agreement is obtained for reverse bias, but the simulated dark current is significantly overestimated for positive bias, and the chosen minority carrier lifetime is anyway very different from the one measured by TRPL. It is thus very unlikely that the large difference observed between the simulation and the experiment originates from the minority carrier lifetime value taken for the simulation.

To go further in the dark current simulation, the model has been refined by adding an ohmic current due to the shunt resistance $\left(\mathrm{J}_{\text {shunt }}\right)$ originating from the surface and bulk leakage currents. The total dark current of the diode $\left(\mathrm{J}_{\text {tot }}\right)$ is then given by

$$
\mathrm{J}_{\text {tot }}=\mathrm{J}_{\text {diode }}+\mathrm{J}_{\text {shunt }} .
$$

The parameters to be determined are the minority carrier lifetime and the $\mathrm{R}_{\text {shunt }}$. A product (with $\mathrm{A}$ the photodiode area). The best agreement with experimental J-V curves

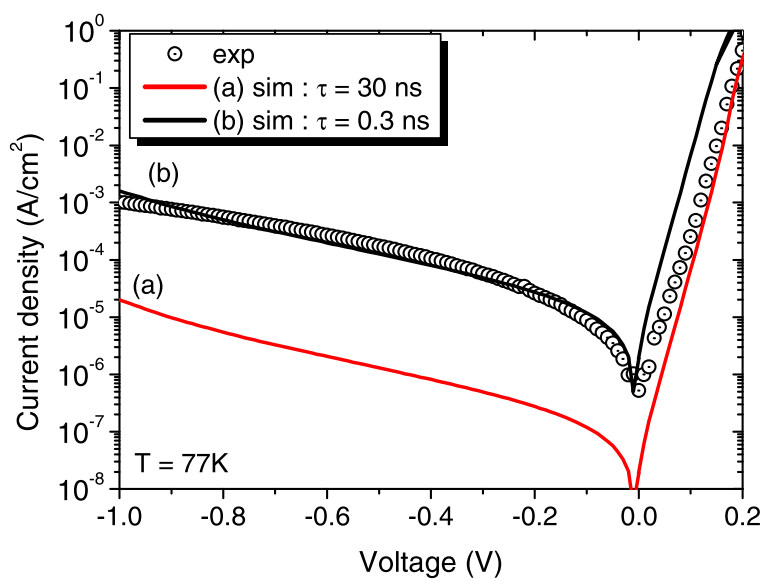

FIG. 4. Experimental (open circles) and simulated (solid line) dark current densities of sample $\mathrm{A}$ at $77 \mathrm{~K}$ with a lifetime value fixed at $30 \mathrm{~ns}$ (a) and at $0.3 \mathrm{~ns}(\mathrm{~b})$. 
across the entire voltage sweep (Figure 5) was obtained for a lifetime of $7 \mathrm{~ns}$ and an $\mathrm{R}_{\text {shunt }}$. A value of $15 \mathrm{k} \Omega \mathrm{cm}^{2}$. Despite the use of the $\mathrm{R}_{\text {shunt }}$, a minority carrier lifetime far from the values measured by TRPL has to be used. Moreover, this fitting method has been applied to a set of different GaSb-rich devices (not described here) having different cut-off wavelengths in the 4.6-5.4 $\mu \mathrm{m}$ range, for which we verified that $\mathrm{R}_{\text {shunt }}$ could not be linked to either the bandgap or the material quality. As the $\mathrm{R}_{\text {shunt }}$ is generally associated with defects in material, as recently shown in MCT photodiodes, ${ }^{29}$ we can conclude that the discrepancy observed between the experimental and simulated curves cannot be ascribed to an additional $\mathrm{R}_{\text {shunt }}$ that would be specific to GaSb-rich photodiodes.

In order to determine the origin of this ohmic current, we studied the photoresponse spectra of the GaSb-rich SL photodiode under an applied electric field.

\section{SPECTRAL RESPONSE UNDER ELECTRIC FIELD}

Spectral response measurements were performed at $80 \mathrm{~K}$ on two GaSb-rich SL photodiodes having different absorption thicknesses $\left(\mathrm{t}_{\mathrm{AZ}}\right): 500 \mathrm{~nm}\left(\mathrm{t}_{\mathrm{AZ} 1}\right)$ (sample $\left.\mathrm{A}\right)$ and $1 \mu \mathrm{m}$ $\left(t_{\mathrm{AZ} 2}\right)$. The curves obtained at different reverse biases have been normalized and plotted in Figure 6.

When a reverse bias is applied, the shape of the spectral response is changing for both samples. We can easily observe in Figure 6 that when the reverse bias is increased, the cut-off wavelength around $4.75 \mu \mathrm{m}$ is red shifted, while the peak around $3.5 \mu \mathrm{m}$ is blue shifted. We assume that the changes of the spectral response shape arise from two valence to conduction inter-miniband transitions, which we denote E1 and E2. The E1 transition corresponds to the fundamental transition between the first heavy hole miniband ( $\mathrm{HH} 1)$ and the first electron miniband $(\mathrm{C} 1)$, while the $\mathrm{E} 2$ transition corresponds to the transition between the second heavy holes miniband (HH2) and C1. When an electric field is applied, these transitions are divided into a "blue" and a "red" component (E1B; $\mathrm{E} 1 \mathrm{R})$ and (E2B; E2R). At an equivalent electric field, the spectral response is essentially the same for both samples, which means that the changes observed originate from the depletion region in the photodiode.

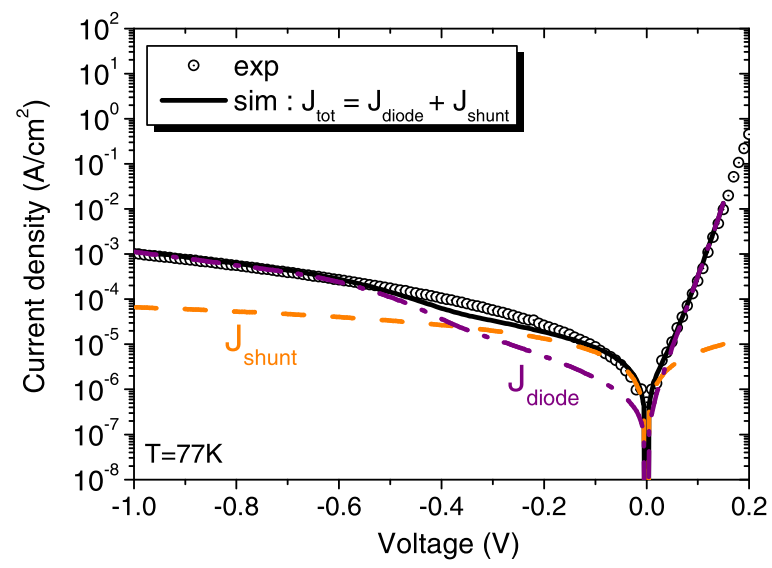

FIG. 5. Experimental (open circles) and simulated (solid line) dark current of sample A at $77 \mathrm{~K}$. The diode current (dashed line) and the ohmic current (dashed-dotted line) are also plotted.
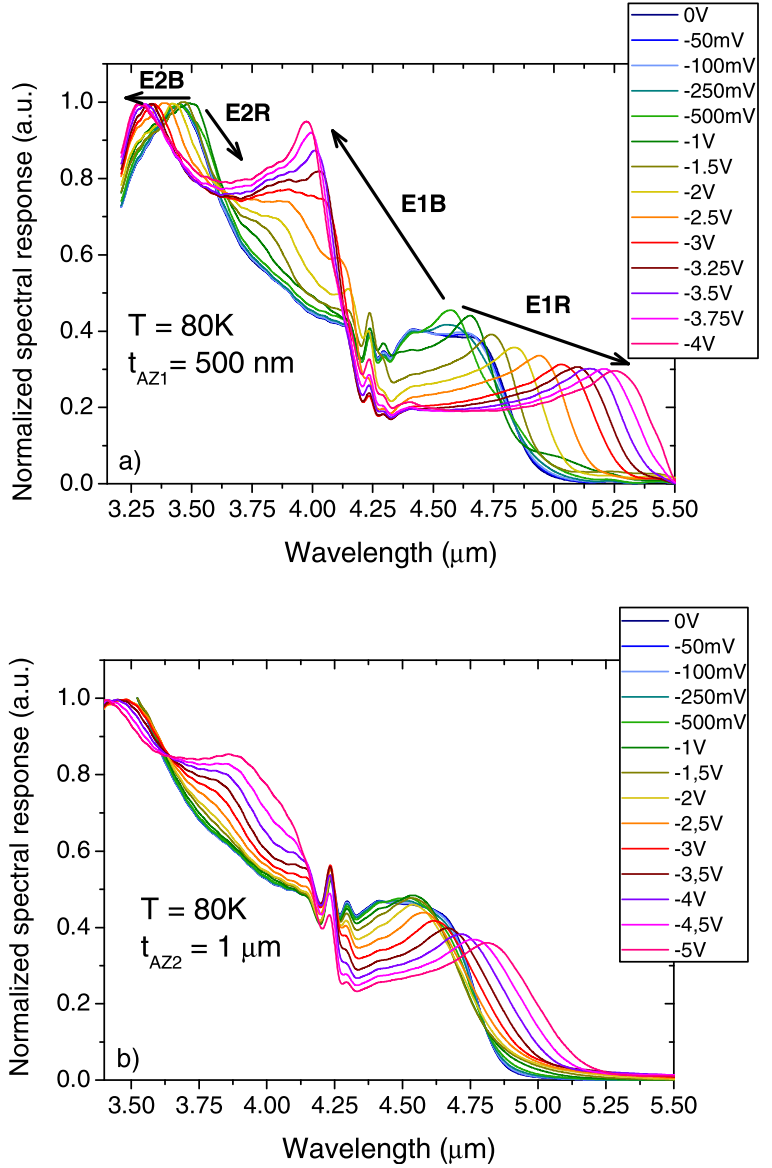

FIG. 6. Photoresponse spectra of reverse biased GaSb-rich SL p-i-n photodiodes measured at $80 \mathrm{~K}$. The absorption thickness is $500 \mathrm{~nm}$ (a) and $1 \mu \mathrm{m}$ (b). Under an applied bias, the interminiband transitions E1 and E2 are divided in two components (E1R; E1B) and (E2R; E2B).

The energy shifts of the SL photoresponse spectra can be attributed to transition energies due to Wannier-Stark localization. Such Stark effects have already been observed in type-II InAs/GaSb SL as changes in both the spectral response shape ${ }^{30}$ and the current-voltage characteristics. ${ }^{30,31}$

In the absence of an electric field, the carriers in the SL are confined in the minibands. When an electric field is applied parallel to the growth direction of the SL structure, the carriers tend to be localized on discrete energy levels, decreasing the spatial coherence of the wavefuntions. Thereby, each miniband is divided into discrete energy levels separated by an energy $\Delta E=q . F . d_{S L}$, where $q$ is the electron charge, $F$ is the electric field, and $d_{S L}$ is the SL period. Stark transitions can therefore occur between valence and conduction energy levels. These transition energies $\mathrm{E}$ are given $b^{30}$

$$
E=E_{0}+m q F d_{S L},
$$

where $E_{0}$ is the transition energy when no electric field is applied and $m$ is the Stark index. In a type-II SL, the transitions being spatially indirect compared to a type-I SL, the Stark indices thus assume the values $m= \pm 1 / 2, \pm 3 / 2, \pm 5 / 2, \ldots$

The carriers are completely localized (electrons and holes in the InAs and the GaSb layers, respectively), when 
the electric field reaches the limit $F_{\text {lim }} \gg \Delta / q d_{S L}$ with $\Delta$ the miniband width. In the case of sample A with $\mathrm{t}_{\mathrm{ZA} 1}=500 \mathrm{~nm}$, we calculated the maximum electric field $F_{\max }$ of the junction as a function of the applied reverse bias, using the following equation:

$$
F_{\max }(V)=\frac{V_{d}-V}{W_{d e p}(V)}
$$

where $V$ is the applied bias, $V_{d}$ is the built-in voltage, and $W_{\text {dep }}$ the depletion width. The result is plotted in Figure 7.

The built-in electric field $F_{0}$ calculated at $0 \mathrm{~V}$ is equal to $8 \mathrm{kV} / \mathrm{cm}$. For the GaSb-SL structure, the electron miniband width being equal to $9 \mathrm{meV}$ and the SL period, measured by HRXD, being equal to $8.9 \mathrm{~nm}$, the electric field $F_{\text {lim }}$ is thus equal to $10 \mathrm{kV} / \mathrm{cm}$. This electric field value is reached when the reverse bias value is only equal to $-135 \mathrm{mV}$ (Figure 7). Thereby, the changes of the spectral response are clearly visible when the reverse applied bias is higher than $135 \mathrm{mV}$ (Figure 6(a)).

To confirm the Wannier-Stark localization in GaSb-rich SL structure, the peak positions E1R and E1B of the spectral response are plotted as a function of the electric field $F$ in the structure (Figure 8), which is equal to the sum of the applied electric field $F_{a p p}$ and the built-in electric field $F_{0}$. The calculated transition energies using the envelope function approximation are also reported. As we can see, the calculated transition energies match quite well with those of the measured spectral response, except for E1B, but this transition is hidden by the $\mathrm{CO}_{2}$ absorption peak around $4.25 \mu \mathrm{m}$ and the transition energies E1B and E2R are probably mixed (Figure 6(a)). Besides that, as expected, the transition energies vary linearly with the electric field and the slopes of the transition E1R and E1B are equal to $-1 / 2$ and $1 / 2$, respectively. This result confirms the formation of localized Wannier-Stark states in the depletion region of the Type-II GaSb-rich SL diode.

A schematic view of the SL p-i-n band diagram is plotted in Figure 9 for an electric field of $18 \mathrm{kV} / \mathrm{cm}$. This electric field value corresponds to an applied reverse bias of $50 \mathrm{mV}$

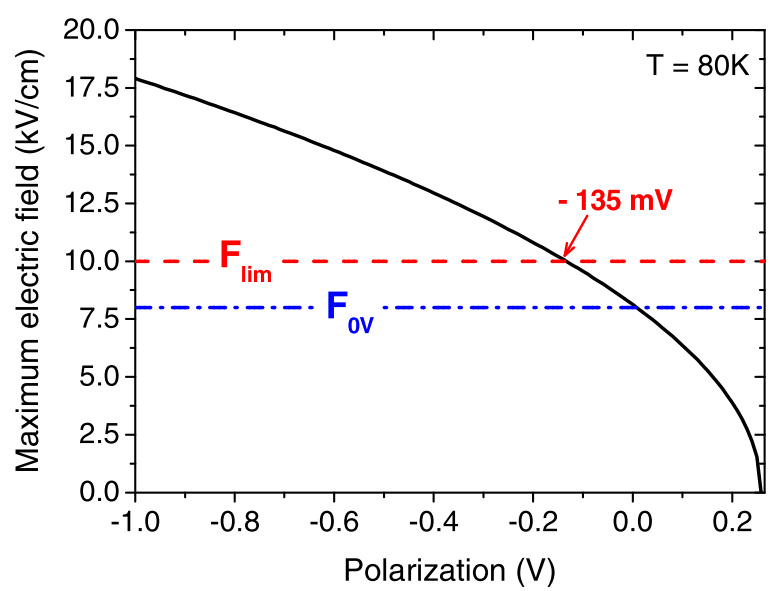

FIG. 7. Maximum electric field of sample A calculated as a function of the applied voltage at $80 \mathrm{~K}$.

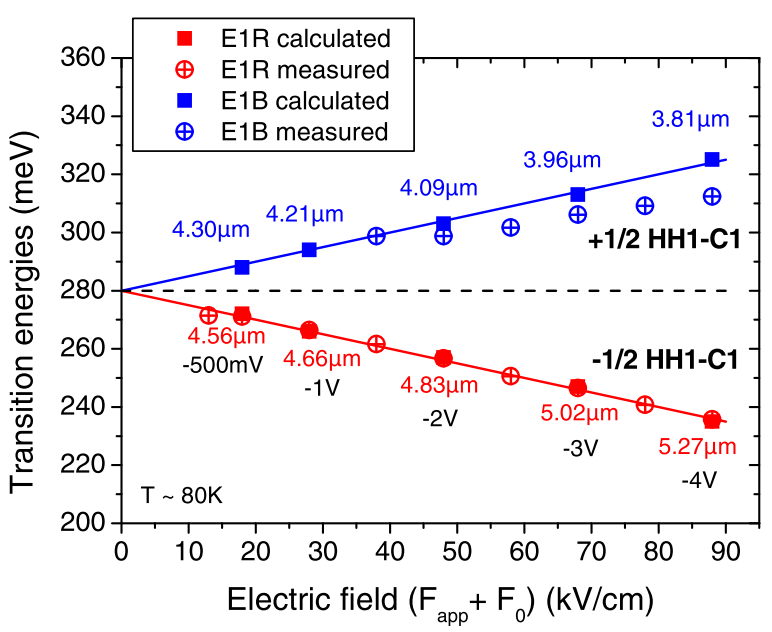

FIG. 8. Transition energies E1R and E1B as a function of the total electric field $F=F_{a p p}+F_{0}$, where $F_{a p p}$ is the applied electric field and $F_{0}$ is the built-in electric field.

on an absorption thickness of $500 \mathrm{~nm}$ with a built-in electric field of $8 \mathrm{kV} / \mathrm{cm}$. The probability densities associated with two electron energy levels surrounding a hole energy level for the transitions E1B and E1R are also plotted. It can be seen that the electric field breaks the three dimensional nature of the InAs/GaSb SL minibands. The carriers are then strongly confined in quasi-bidimensional states, just like in an InAs/GaSb multi-quantum well structure, with electrons and holes localized in InAs and GaSb layers, respectively.

Because the minibands are reduced into discrete energy levels, a tunneling current can therefore appear in the case of $\mathrm{p}$-i-n photodiodes. This tunneling current occurs between the top of the first hole miniband $\mathrm{HH} 1$ of the P-region and a discrete energy level which is energetically aligned in the intrinsic region of the photodiode, as suggested by Bürkle et $a .^{30}$ and as illustrated in Figure 10. It is thus obvious that the simulation previously described cannot take into account this strong discontinuity of the SL. The ohmic current used to adjust the simulation to the experiment is probably linked to this tunneling current into the GaSb-rich p-i-n photodiode. Additionally, this tunneling current may also explain why we did not see significant improvements of the dark current

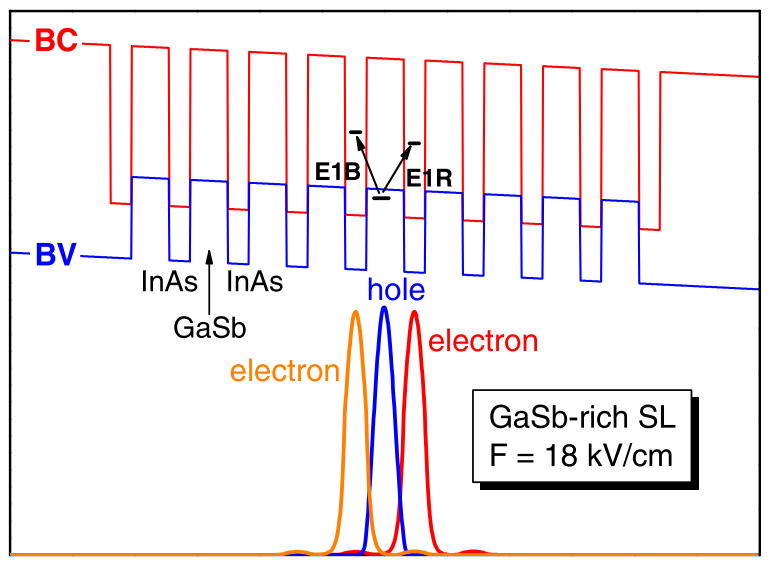

FIG. 9. Band diagram of the GaSb-rich SL at an applied electric field of $18 \mathrm{kV} / \mathrm{cm}$. The electron and hole probability densities for the E1B and E1R transitions are also shown. 


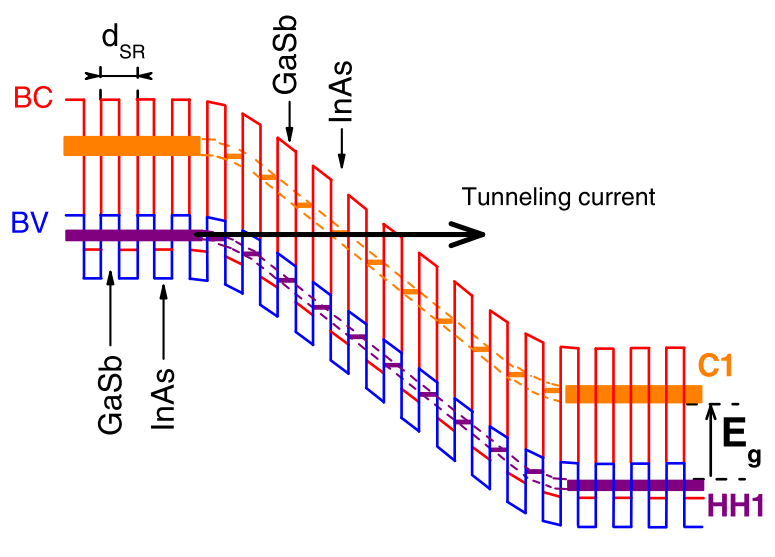

p

i

n

FIG. 10. Schematic view of the GaSb-rich SL p-i-n photodiode. ${ }^{30}$

performance when changing the growth conditions of the GaSb-rich SL structure.

In the case of InAs-rich SL, we did not observe the Wannier-Stark effect on the spectral response. Indeed, the carriers are completely delocalized even if an electric field is applied. The carrier are completely localized when the electric field reaches an estimated value of $1.7 \times 10^{3} \mathrm{kV} / \mathrm{cm}$, which is three orders of magnitude superior than in the case of the GaSb-rich SL. The InA-rich SL structure, given its thin period $(3.3 \mathrm{~nm})$ and its large conduction miniband, is not disturbed by the electric field, regardless of the applied bias.

\section{CONCLUSION}

In conclusion, we studied the GaSb-rich SL MWIR photodiodes which have a higher current density than the InAs-rich SL ones. Alternative growth conditions were explored in order to improve the material quality and thus improve the minority carrier lifetime in the GaSb-rich SL structure. However, upon improving the material quality, the dark currents obtained were still of the same order of magnitude. It seems that an intrinsic phenomenon in the GaSb-rich SL structures is deteriorating the performance; this intrinsic phenomenon has been modeled using an ohmic current in the dark current simulations. By analyzing the spectral response, we have clearly identified that the behavior of the GaSb-rich SL structures depends on the electric field in the active region and also on the periodicity of the SL structure. This dependence is characterized by the apparition of spectral signatures in the photoresponse attributable to Wannier-Stark ladder. Besides the presence of defects inherent in a GaSb-rich structure, the electric field induces a strong tunneling current at low biases, which therefore limits the current-voltage characteristics. This limiting mechanism can explain the high current density of the GaSb-rich SL photodiode. Nevertheless, at the present state of our theoretical investigations, the quantum effects into the GaSb-rich SL structures and their influence on the dark current are difficult to quantify, and an accurate transport model is required to explain the performance of $\mathrm{GaSb}$ rich SL p-i-n photodiodes.

\section{ACKNOWLEDGMENTS}

This work was partially funded by the French DGA and by the French "Investment for the Future" program (EquipEx EXTRA, ANR 11-EQPX-0016).

${ }^{1}$ M. Razeghi, A. Haddadi, A. M. Hoang, G. Chen, S. Bogdanov, S. R. Darvish, F. Callewaert, P. R. Bijjam, and R. Mc Clintock, "Antimonidebased type II superlattices: A superior candidate for the third generation of infrared imaging systems," J. Electron. Mater. 43, 2802 (2014).

${ }^{2}$ N. Gautam, M. Naydenkov, S. Myers, A. V. Barve, E. Plis, T. Rotter, L. R. Dawson, and S. Krishna, "Three color infrared detector using InAs/ GaSb superlattices with unipolar barriers," Appl. Phys. Lett. 98, 121106 (2011).

${ }^{3}$ C. H. Grein, P. M. Young, and H. Ehrenreich, "Minority carrier lifetimes in ideal InGaSb/InAs superlattices,” Appl. Phys. Lett. 61, 2905 (1992).

${ }^{4}$ D. R. Rhiger, "Performance comparison of long-wavelength infrared type II superlattice devices with HgCdTe," J. Electron. Mater. 40, 1815 (2011).

${ }^{5}$ E. A. Plis, "InAs/GaSb Type-II superlattice detectors," Adv. Electron. 2014, 246769.

${ }^{6}$ D. Donetsky, S. P. Svensson, L. E. Vorobjev, and G. Belenky, "Carrier lifetime measurements in short-period InAs/GaSb strained-layer superlattice structures," Appl. Phys. Lett. 95, 212104 (2009).

${ }^{7}$ L. M. Murray, K. S. Lokovic, B. V. Olson, A. Yildirim, T. F. Boggess, and J. P. Prineas, "Effects of growth rate variations on carrier lifetime and interface structure in InAs/GaSb superlattices," J. Cryst. Growth 386, 194 (2014).

${ }^{8}$ B. C. Connelly, G D. Metcalfe, H. Shen, and M. Wraback, "Direct minority carrier lifetime measurements and recombination mechanisms in longwave infrared type II superlattices using time-resolved photoluminescence," Appl. Phys. Lett. 97, 251117 (2010).

${ }^{9}$ D. Donetsky, G. Belenky, S. Svensson, and S. Suchalkin, "Minority carrier lifetime in type-2 InAs-GaSb strained-layer superlattices and bulk HgCdTe materials," Appl. Phys. Lett. 97, 052108 (2010).

${ }^{10}$ S. P. Svensson, D. Donetsky, D. Wang, H. Hier, F. J. Crowne, and G. Belenky, "Growth of type II strained layer superlattice, bulk InAs and GaSb materials for minority lifetime characterization," J. Cryst. Growth 334, 103 (2011).

${ }^{11}$ B. C. Connelly, G. D. Metcalfe, H. Shen, M. Wraback, C. L. Canedy, I. Vurgaftman, J. S. Melinger, C. A. Affouda, E. M. Jackson, J. A. Nolde, J. R. Meyer, and E. H. Aifer, "Investigation of trap states in mid-wavelength infrared type II superlattices using time-resolved photoluminescence," J. Electron. Mater. 42, 3203 (2013).

${ }^{12}$ B. Klein, N. Gautam, E. Plis, T. Schuler-Sandy, T. J. Rotter, and S. Krishna, "Carrier lifetime studies in midwave infrared type-II InAs/GaSb strained layer superlattice,” J. Vac. Sci. Technol. B 32, 02C101 (2014).

${ }^{13}$ J. Pellegrino and R. DeWames, "Minority carrier lifetime characteristics in type-II InAs/GaSb LWIR superlattice $\mathrm{n}^{+} \pi \mathrm{p}^{+}$photodiodes," Proc. SPIE 7298, $72981 \mathrm{U}$ (2009).

${ }^{14}$ D. Zuo, P. Qiao, D. Wasserman, and S. L. Chuang, "Direct observation of minority carrier lifetime improvement in InAs/GaSb type II superlattice photodiodes via interfacial layer control,” Appl. Phys. Lett. 102, 141107 (2013).

${ }^{15}$ B. V. Olson, E. A. Shaner, J. K. Kim, J. F. Klem, S. D. Hawkins, L. M. Murray, J. P. Prineas, M. E. Flatté, and T. F. Boggess, “Time-resolved optical measurements of minority carrier recombination in a mid-wave infrared InAsSb alloy and InAs/InAsSb superlattice,” Appl. Phys. Lett. 101, 092109 (2012).

${ }^{16}$ E. H. Steenbergen, B. C. Connelly, G. D. Metcalfe, H. Shen, M. Wraback, D. Lubyshev, Y. Qiu, J. M. Fastenau, A. W. K. Liu, S. Elhamri, O. O. Cellek, and Y.-H. Zhang, "Significantly improved minority carrier lifetime observed in a long-wavelength infrared III-V type-II superlattice comprised of InAs/InAsSb,” Appl. Phys. Lett. 99, 251110 (2011).

${ }^{17}$ T. Schuler-Sandy, S. Myers, B. Klein, N. Gautam, P. Ahirwar, Z.-B. Tian, T. Rotter, G. Balakrishnan, E. Plis, and S. Krishna, "Gallium free type II InAs/InAs $\times$ Sb 1-x superlattice photodetectors," Appl. Phys. Lett. 101, 071111 (2012).

${ }^{18}$ P. C. Klipstein, Y. Livneh, A. Glozman, S. Grossman, O. Klin, N. Snapi, and E. Weiss, "Modeling InAs/GaSb and InAs/InAsSb superlattice infrared detectors," J. Electron. Mater. 43, 2984 (2014).

${ }^{19}$ R. Taalat, J. B. Rodriguez, M. Delmas, and P. Christol, "Influence of the period thickness and composition on the electro-optical properties of 
type-II InAs/GaSb midwave infrared superlattice photodetectors," J. Phys. D: Appl. Phys. 47, 015101 (2014).

${ }^{20}$ E. Giard, I. Ribet-Mohamed, J. Jaeck, T. Viale, R. Haïdar, R. Taalat, M. Delmas, J. B. Rodriguez, E. Steveler, N. Bardou, F. Boulard, and P. Christol, "Quantum efficiency investigations of type-II InAs/GaSb midwave infrared superlattice photodetectors," J. Appl. Phys. 116, 043101 (2014).

${ }^{21}$ J. B. Rodriguez, P. Christol, L. Cerutti, F. Chevrier, and A. Joullié, "MBE growth and characterization of type-II InAs/GaSb superlattices for midinfrared detection," J. Cryst. Growth 274, 6 (2005).

${ }^{22}$ R. Chaghi, C. Cervera, H. Aï-Kaci, P. Grech, J. B. Rodriguez, and P. Christol, "Wet etching and chemical polishing of InAs/GaSb superlattice photodiodes," Semicond. Sci. Technol. 24, 065010 (2009).

${ }^{23}$ M. Delmas, J. B. Rodriguez, and P. Christol, "Electrical modeling of InAs/ GaSb superlattice mid-wavelength infrared pin photodiode to analyze experimental dark current characteristics," J. Appl. Phys. 116, 113101 (2014).

${ }^{24}$ B. M. Nguyen, D. Hoffman, P. Y. Delaunay, E.-K. Huang, and M. Razeghi, "Very high performance LWIR and VLWIR Type-II InAs/GaSb superlattice photodiodes with M-structure barrier," Proc. SPIE 7082, 708205 (2008).
${ }^{25}$ See https://dynamic.silvaco.com/dynamicweb/jsp/downloads/Download ManualsAction.do?req=silen-manuals\&name=atlas for Silvaco ATLAS Users Manual.

${ }^{26}$ I. Vurgaftman, J. R. Meyer, and L. R. Ram-Mohan, "Band parameters for III-V compound semiconductors and their alloys," J. Appl. Phys. 89, 5815 (2001).

${ }^{27}$ M. Delmas, J. B. Rodriguez, R. Taalat, L. Konczewicz, W. Desrat, S. Contreras, E. Giard, I. Ribet-Mohamed, and P. Christol, "Midwave infrared InAs/GaSb superlattice photodiode with a dopant-free pn junction," Infrared Phys. Technol. 70, 76 (2015).

${ }^{28}$ G. A. M. Hurkx, H. C. Degraff, W. J. Kloosterman, and M. P. G. Knuvers, "A new analytical diode model including tunneling and avalanche breakdown," IEEE Trans. Electron Devices 39, 2090 (1992).

${ }^{29} \mathrm{~V}$. Gopal, "A new approach to investigate leakage current mechanisms in infrared photodiodes from illuminated current-voltage characteristics," J. Appl. Phys. 116, 084502 (2014).

${ }^{30}$ L. Bürkle, F. Fuchs, E. Ahlswede, W. Pletschen, and J. Schmitz, "Wannier-Stark localization in InAs/GAInSb superlattice diodes," Phys. Rev. B 64, 045315 (2001)

${ }^{31}$ Y. Wei, A. Hood, H. Yau, A. Gin, M. Razeghi, M. Z. Tidrow, and V. Nathan, "Uncooled operation of type-II InAs/GaSb superlattice photodiodes in the midwavelength infrared range," Appl. Phys. Lett. 86, 233106 (2005). 